\title{
Efficient Interactive Multicast over DVB-T2 - Utilizing Dynamic SFNs and PARPS
}

\author{
Magnus Eriksson, S.M. Hasibur Rahman, Francisco Fraile and Mårten Sjöström
}

\begin{abstract}
In the terrestrial digital TV systems DVB-T/H/T2, broadcasting is employed, meaning that all TV programs are sent over all transmitters, also where there are no viewers. This is inefficient utilization of spectrum and transmitter equipment. Applying interactive multicasting over DVB-T2 is a novel approach that would substantially reduce the spectrum required to deliver a certain amount of TV programs. Further gain would be achieved by Dynamic single-frequency network (DSFN) formations, which can be implemented using the concept of PARPS (Packet and Resource Plan Scheduling). A Zipf-law heterogeneous program selection model is suggested. For a system of four coordinated transmitters, and certain assumptions, IP multicasting over non-continuous transmission DSFN gives $1740 \%$ increase in multiuser system spectral efficiency (MSSE) in (users·bit/s)/Hz/site as compared to broadcasting over SFN.
\end{abstract}

Index Terms-Digital Video Broadcasting, IP Multicast, DSFN, LSA, IP-TV, Mobile-TV, Zipf, DVB-T2, eMBMS, MBSFN, cooperative macro-diversity.

\section{INTRODUCTION}

I NTERACTIVE multicast allows efficient transmission of streaming services over a multi-point channel, by only delivering content to viewers that have sent a join message over an interaction channel. As an example, IP multicast is the foundation of wired IPTV, because it saves valuable bandwidth as compared to broadcast or unicast. Moreover, switched broadcast is an upcoming cable TV network technology that resembles a multicast service, where only the currently most popular content is delivered [1]. The operator offers a number of services higher than the maximum allowed in the network, assuming that it is unlikely that all of the offered TV programs (i.e. MPEG transport streams) are requested at the same time in a certain area. The concept of interactive multicast allows more $\mathrm{TV}$ services to be

Extended abstract received December 26, 2012

M. Eriksson. Author is with the ITM department at the Mid Sweden University and currently working as lecturer, SWEDEN. (Phone: 004670-562 5502; fax: 004660-148830, e-mail: magnus.eriksson@miun.se).

S.M. H. Rahman. Author is with the ITM department at the Mid Sweden University and currently working as a research assistant, SWEDEN (Phone: 004670-746-3968, e-mail: hasibur.rahman@miun.se).

F. Fraile. Author is with the Universitat Politècnica de València, SPAIN. (e-mail: ffraile@iteam.upv.es).

Prof. M. Sjöstrom. Author is with the Mid Sweden University, 3D research group.

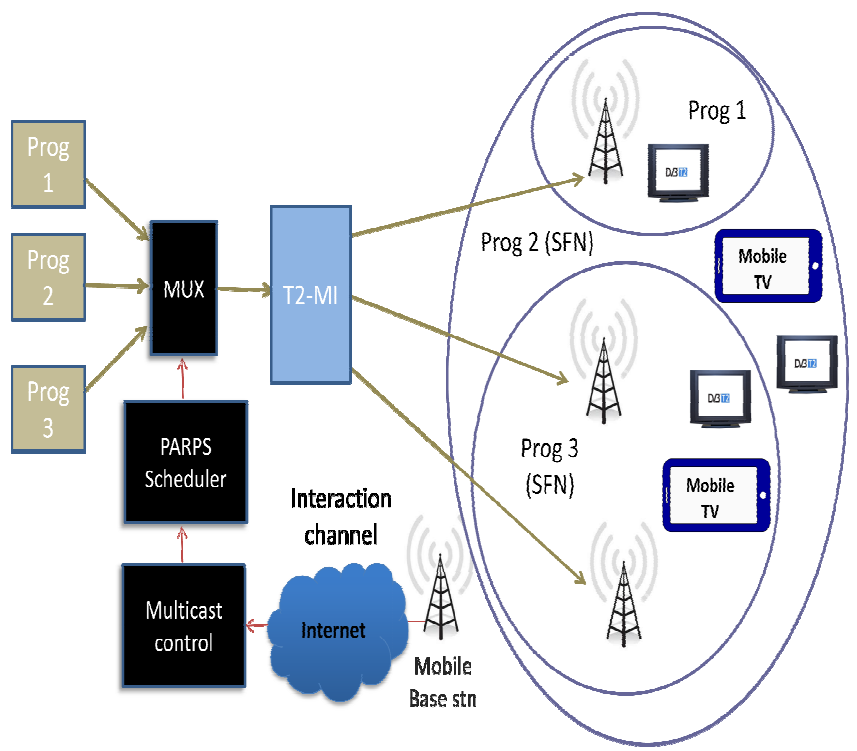

Fig. 1. A coordinated system of three DVB-T2 transmitters may send global content in some timeslots, and local content in others. Multicast join/leave messages, and measurement data may be sent over an interaction channel.

transmitted over a given spectrum, at the expense of an interaction channel and a blocking probability (the probability that the number of currently requested TV programs exceeds the system capacity).

A novel topic of this paper is to investigate the improved efficiency of interactive multicast schemes in terrestrial TV, something that to our knowledge not has been offered before.

Multicasting can increase the system spectral efficiency (SSE) in bit/s/Hz/transmitter, i.e. reduce the required spectrum, or it can increase the number of TV programs that can be offered over a given spectrum. It is especially of interest for mobile-TV, since an interaction channel is already available.

A second novelty of this paper is the introduction of a heterogeneous user behavior model, representing two distinct groups of users living in different parts of the area, with different TV program preferences. Multicasting can take advantage of heterogeneous TV program selection.

A third novelty is a new use of the packet and resource plan scheduling (PARPS) scheme, which was introduced in 1999, at that time for unicast services.[2] In this study, the scheme is extended to multicast services. PARPS provides a generic and optimized approach to packet-by-packet dynamic radio resource management (RRM).

In this paper, PARPS is used as an approach to Dynamic 


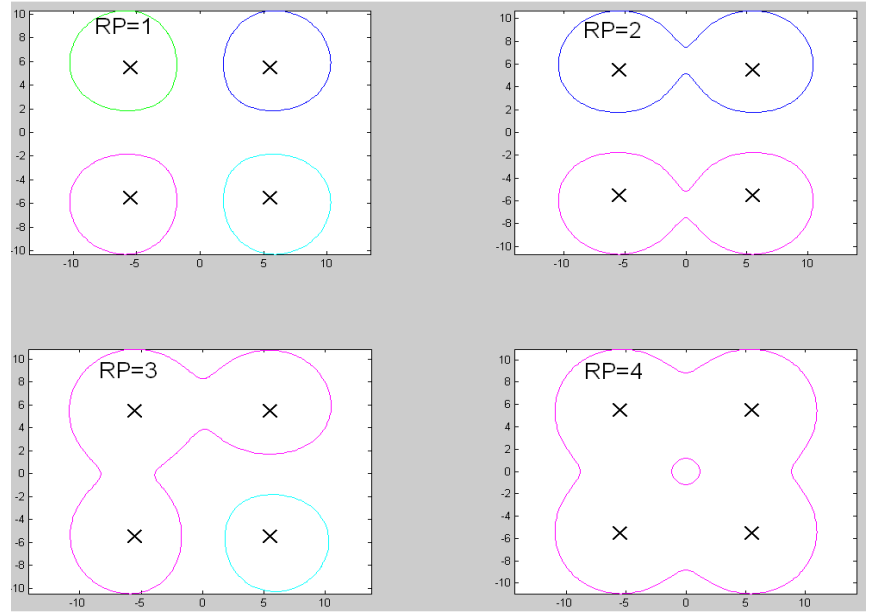

Fig. 2. Simple Continuous Transmission DSFN case with no fading, for four transmitters (x) and four resource plans (RP). The calculated coverage of each zone has different color. No fading.

Single Frequency Networks (DSFNs). DSFN is the dynamic formation of Single-Frequency Networks (SFNs), i.e groups of transmitters sending the same signal simultaneously over the same frequency; something facilitated by OFDM and other frequency-domain equalization schemes. In DSFN schemes, the SFN grouping is changed dynamically from timeslot to timeslot by a scheduling scheme, based on traffic load and channel condition measurements. It facilitates efficient implementation of interactive multicast over terrestrial DVB, and may further increase the system spectral efficiency (SSE).

The expense of DSFN is receiver side channel condition measurements, to be sent over the interaction channel. DSFN was studied for the unicast interactive service in our previous work ([2], [3]), where it had similar aim as soft-handover in 3G cellular systems, and Coordinated Multi-point Transmission (COMP) in the LTE 4G system.

Fig. 2 illustrates PARPS for a system consisting of four transmitters, all using the same frequency channel. Four alternative resource plans are defined, i.e. four different ways to group the base station transmitters into SFN:s.

During timeslots that are assigned to resource plan 1, each transmitter belongs to its own SFN of size one transmitter, each sending different signals. The coverage area of each SFN is called a zone, which is limited in this case due to their mutual co-channel interference (cross-talk).

When using resource plan 2 and 3, transmitters form bigger zones which gives better coverage. However, with two zones, only two different signals (two TV programs at a time) can be delivered simultaneously in the system. During these timeslots, the SSE in bit/s/Hz/transmitter is half the SSE of resource plan 1 .

When selecting the resource plan 4 , all the transmitters are grouped together into one SFN, and the coverage is only limited by noise and interference from external transmitters. This results in a larger coverage area of the system. However, since only one TV program at a time can be sent, the SSE would suffer if plan 1 is used often. The most popular programs should be transmitted using this resource plan, thus serving many users and resulting in what we here call a high multi-user SSE (MSSE) in users.bit/s/Hz/transmitter.

The duty of the PARPS scheme is to assign one resource plan to each timeslot in the centrally controlled system, and to assign data to each zone during that timeslot, based on channel condition measurements and current traffic load. The objective function of the scheduling is to maximize the MSSE.

A data packet queue is arranged for each zone in each resource plan. An incoming data packet addressed to a certain multicast group is placed in several queues, one for each resource plan that it can be covered by. Based on an objective function, such as maximizing the throughput, the scheme assigns one resource plan to each timeslot in the zone. This concept allows dynamic RRM for each data packet without the need of calculating traffic-dependent signal-to-interferenceand-noise ratio (SINR), but only once for each user in each resource plan, as long as channel conditions do not change. PARPS is a generic approach, which also may be used for timeslot-by-timeslot control of RRM parameters such as transmission power, link adaptation, MIMO, and many other RRM parameters.

\section{IMPLEMENTATION ASPECTS}

Fig 2 is an example of continuous transmission DSFN (CTDSFN), where transmitters send with constant power, while in non-continuous transmission DSFN (NCT-DSFN) the signal is switched off in certain resource plans, meaning it is keyed or switched on and off between timeslots. NCT-DSFN is however not practically feasible without modification of today's DVB-T/T2 transmitter equipment. In current transmitters, automatic power control is designed to provide a constant non-zero radiated power level. When the input signal to the transmitter is shut of, the power control fails at achieving the desired level and triggers a failure signal.

On the other hand, DSFN also requires that today's statistical multiplexors are replaced by more advanced scheduling equipment.

A concept similar to DSFNs was introduced in [4] and [5], for the insertion of local broadcast content in DVB-H and DVB-T MUX streams, covering local service areas (LSA), i.e. stationary SFN formations.

DSFN requires introduction of time slots, and would therefore benefit from new features of the DVB-T2 standard, such as Time-Frequency Slicing (TFS) and local service insertion through the DVB-T2 Modulator Interface (T2-MI). Time slicing is utilized in DVB-T2 to provide dynamic allocation of services. See Fig 1

Return channels such as DVB-RCT have been discussed in several papers, and interactive services have been tested in pilot projects such as Multimedia-Home-Platform,[11] but without a clear killer application. However, return channels are available for mobile-TV reception, and also increasingly in Internet connected TV sets. We suggest that interactive multicast as a major application of a return channel.

The combination of DSFN and interactive multicasting resembles the eMBMS cellular service (enhanced Multimedia Broadcast Multicast Service) for cellular 4G LTE networks, 
where SFNs are known as multimedia broadcast single frequency networks (MBSFNs) [6]. PARPS and NCT-DSFN can potentially be utilized to achieve optimized eMBMS transmission, with Multicast-Broadcast Single Frequency Network (MB-SFN) channels. The resource plans should then also control other RRM parameters such as transmission power, error coding, MIMO, etc.

\section{COMPARED SCHEMES}

A total of seven schemes have been designed and analyzed in terms of coverage probability and multiuser system spectral efficiency in different scenarios. Scheme A and $\mathrm{C}$ are reference schemes, representing today's conventional multifrequency network (MFN, i.e. non-SFN) and single frequency networks (SFN), while the others are suggested schemes. Combinations of multicasting, and DSFN, with and without continous transmission (CT-DSFN or NCT-DSFN), have been studied.

\section{- $\quad$ Scheme A: Broadcasting over MFN (used in today's DVB-T/T2/H) \\ - Scheme B: Multicasting over MFN (similar to basic MBMS and eMBMS) \\ - Scheme C: Broadcasting over SFN (used in some of today's DVB-T/T2/H networks) \\ - Scheme D: Multicasting over SFN \\ - Scheme E: Multicasting over CT-DSFN \\ - $\quad$ Scheme F: Multicasting over non-SFN NCT (Dynamic channel allocation on a packet-by-packet basis) \\ - $\quad$ Scheme G: Multicasting over NCT-DSFN}

In scheme E, F and G, PARPS is utilized, where each scheme corresponds to a certain set of resource plans.

\section{MODELS AND PERFORMANCE MEASURES}

The simulation parameters are presented in Table I.

\section{A. Program selection models}

Zipf-law user behavior is assumed, where the most popular TV program corresponds to rank $r=1$, the second most popular program rank $r=2$, etc, and the number of offered programs is $N_{\text {pro. }}$ The probability that an active user has chosen a TV program of rank $r$ is: [7]

$$
\mathrm{f}_{\mathrm{r}}=\frac{1 / \mathrm{r}^{\theta}}{\sum_{n=1}^{\mathrm{N} p \mathrm{o}} 1 / \mathrm{n}^{\theta}}
$$

A heterogeneous user behavior model is suggested, where different programs are popular in different parts of the system. Users in the left half of the simulated area have one ranking order (what program that corresponds to rank 1, 2, etc, in the Zipf distribution), and users on the right side another, independently of each other, based on a random permutation.

Results are also provided for a conventional homogeneous program selection model, where the Zipf-law program
TABLE I

SIMULATION PARAMETERS

\begin{tabular}{llc}
\hline \hline Symbol & Quantity & Value \\
\hline$N_{\mathrm{Tx}}$ & number of transmitters & 4 \\
$N_{\mathrm{Rx}}$ & number of receivers & $100^{*}$ \\
$N_{\text {pro }}$ & number of programs & $100^{*}$ \\
SINR $\Gamma$ & Required signal-to-interference-and-noise ratio & $10 \mathrm{~dB}$ \\
& $\quad$ (non-SFN case) & $5 \cdot 10^{-4}$ \\
$G$ & antenna gain & 4 \\
$\alpha$ & propagation path loss exponent & $8 \mathrm{~dB}$ \\
$\sigma$ & log-normal fading standard deviation & 0.95 \\
$\theta$ & zipf exponent & $6 \cdot 10^{-8} \mu \mathrm{W}$ \\
$N$ & external interference and noise level & $6 \mathrm{MHz}$ \\
$B_{C h}$ & channel bandwidth
\end{tabular}

* = varies in different simulations

probabilities are the same in all parts of the region. Real user behavior, in case of two distinct groups of users, is expected to be in between these two extreme models.

\section{B. Single-frequency network signal quality model}

A locally centralized system consists of a set of $N_{\text {Tx }}$ centrally controlled and synchronized base station transmitters, sending information to the set of $N_{\mathrm{Rx}}$ active receiver terminals. The received power from transmitter $i \in \mathrm{TX}=\left\{1,2, \ldots, N_{T x}\right\}$ at receiver $j \in \mathrm{RX}=\left\{1,2, \ldots, N_{R x}\right\}$ is denoted $P_{i, j}$.

An SFN is a set of one or several transmitters sending the same information simultaneously over the same frequency channel. The Signal-to-Interference-and-noise Ratio (SINR) at receiver $j$, averaged over all OFDM sub-carriers, is: [8]

$$
\Gamma_{j}=\frac{\sum_{i \in \mathrm{U}_{j}} P_{i, j} w_{i, j}}{\sum_{i \in \mathrm{I}_{j}} P_{i, j}+\sum_{i \in \mathrm{U}_{j}} P_{i, j}\left(1-w_{i, j}\right)+I_{\mathrm{Ext}, j}} \approx \frac{\sum_{i \in \mathrm{U}_{j}} P_{i, j}}{\sum_{i \in \mathrm{I}_{j}} P_{i, j}+I_{\mathrm{Ext}}},
$$

where $\mathrm{U}_{j} \subseteq \mathrm{TX}$ is the set of transmitters in the SFN assigned

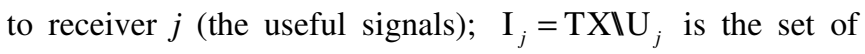
sending transmitters assigned to other receivers (the cochannel interferers) in the centralized system; and $I_{\mathrm{Ext}, j}$ is the external interference and noise power, including thermal noise as well as power from transmitters outside the coordinated system. $w_{i, j} \in[0,1]$ is a weighting factor depending on the OFDM inter-symbol interference (ISI, caused by time spreading) and inter-carrier interference (ICI, caused by Doppler shift). However, we neglect ISI and ICI. This leads to the approximation in the right term of (2).

\section{Wave propagation model}

Random receiver positions, and random log-normal shadow fading, are assumed.

The power $P_{i, j}$ in Watt received at receiver position $j$ from transmitter $i$ is modeled according to the log-distance path loss model (here expressed in non-logarithmic form): [9] 


$$
P_{i, j}=\frac{P_{i} F_{i, j} G_{i, j}}{d_{i, j}^{\alpha}}
$$

Here, $P_{i}$ is the transmitter power sent from transmitter $i, F_{i, j}$ is the random shadow fading effect which is assumed to be log-normal distributed with standard deviation $\sigma, \mathrm{d}_{\mathrm{i}, \mathrm{j}}$ is the distance between receiver $j$ and transmitter $I ; G_{\mathrm{i}, \mathrm{j}}$ is the antenna gain, here assumed to be constant (for omnidirectional antennas); and $\alpha$ is and exponent which would be 2 in free space, with values in table I.

The OFDM modulation is assumed to eliminate the effect of multi-path propagation fading. The effect of mobility is not studied.

\section{Performance Measures}

The Multiuser System Spectral Efficiency (MSSE) in users $\cdot$ bit/s/Hz/transmitter is a metric hereby introduced as a normalized measure of the number of users that are served by multicasting:

$$
S_{M}=\frac{\eta_{M} R_{\mathrm{Ch}}}{B_{\mathrm{Ch}}}
$$

where, $R_{\mathrm{Ch}}$ is the information rate, and $B_{C h}$ is the channel bandwidth in Hz. The multiuser channel utilization $\eta_{M}$ is:

$$
\eta_{M}=\frac{N_{\mathrm{CRx}}}{N_{\mathrm{Tx}} N_{\mathrm{Ch}}}
$$

where, $N_{\mathrm{CRx}}$ is the number of active receivers (or users) being covered, $N_{\text {Tx }}$ is the number of transmitters and $N_{C h}$ is the number of channels (timeslots and frequencies) required to deliver all selected TV programs to the receivers.

The MSSE resembles the conventional System Spectral Efficiency (SSE) measure in bit/s/Hz/transmitter, but reflects number of happy users rather than number of transmitted TV programs in a given spectrum. Popular TV programs have higher weight. If not all selected programs are delivered, but popular programs are prioritized while the number of programs, the spectrum bandwidth and the number of transmitters are kept constant, the MSSE would increase, while the SSE would be constant.

\section{THE PARPS ALGORITHM}

The PARPS scheme was briefly introduced in section I. Some mathematical notation and criteria are provided in this section. For details on the scheduling scheme, and optimization formulation of the scheduling problem, see [3].

The PARPS approach turns dynamic radio resource management into a scheduling scheme, which can be seen as an optimization problem. The objective function may for example be to maximize the MSSE, under constraint that that the signal-to-interference ratio should be sufficient, i.e. $\Gamma_{j}>\gamma_{m}$ for all receivers.

The SIR bound $\gamma_{m}$ for the chosen error correction and modulation scheme $m$ should include a margin for measurement data inaccuracy, e.g. due to terminal motion

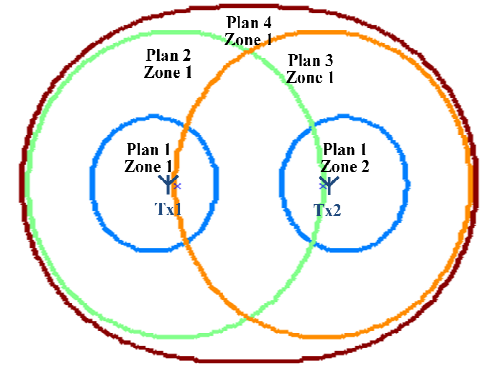

Fig. 3. A simple example: Zone borders for NCT-DSFN with two transmitters and four resource plans. Plan $r=1$ : Both transmitters send different information on the same channel. Plan 2: Only Tx1 sends. Plan 3: Only Tx2 sends. Plan 4: Both transmitters send the same information on the same channel, i.e. they form a Single Frequency Network.

since last measurement. The margin should be based on the measured variance of the SIR, and chosen for a desired bit error probability probability.

The coverage (capture) area of each SFN, such that $\Gamma>\gamma_{m}$, is here called a zone. In this paper, a resource plan is a certain grouping of the base station transmitters into SFN:s.

\section{A. Resource plans}

A transmitter assignment plan $\mathbf{T}^{(r)}$ is a matrix that specifies the transmitter power levels and the assignment of transmitters to zones in resource plan $r$, according to:

$T_{z, i}^{(r)}= \begin{cases}\text { power from transmitter } i, & \text { if it belongs to zone } z \text { of plan } r \\ 0, & \text { otherwise }\end{cases}$

In this study, no power control is practiced, i.e. all transmitters either send with full power $P_{\max }$ or are blocked:

$$
T_{z, i}^{(r)} \in\left\{0, P_{\max }\right\} \quad \forall z, i, r .
$$

Simple example: The example in Fig. 3 is generated from the following set of transmitter assignment plans:

$$
\begin{aligned}
& \mathrm{T}=\left\{\left[\begin{array}{ll}
1 & 0 \\
0 & 1
\end{array}\right],\left[\begin{array}{ll}
1 & 0 \\
0 & 0
\end{array}\right],\left[\begin{array}{ll}
0 & 1 \\
0 & 0
\end{array}\right],\left[\begin{array}{ll}
1 & 1 \\
0 & 0
\end{array}\right]\right\}\left\{\begin{array}{c}
\text { Zone 1 } \\
\text { Zone 2 }
\end{array}\right.
\end{aligned}
$$

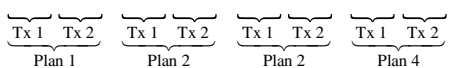

The matrix has $N_{\text {Tx }}$ columns and $N_{\text {Zones }}$ rows, where $N_{\text {Zones }} \leq N_{\text {Tx }}$ is the maximum number of zones in each resource plan. The zone index $z$ is a member of the set $\left\{1,2, \ldots, N_{\text {Zones }}\right\}$.

The resource plan index $r$ belongs to the set RP of $N_{\mathrm{Rp}}$ alternative resource plan indices.

In a valid transmitter assignment plan, maximum one zone is assigned to each transmitter, corresponding to the following criteria: $\quad \sum_{z} \theta\left(T_{z, i}^{(r)}\right) \leq 1, \quad \forall i, r$,

where the Heavyside function $\theta(\cdot)$ returns 1 if the argument is non-zero, otherwise 0 . 


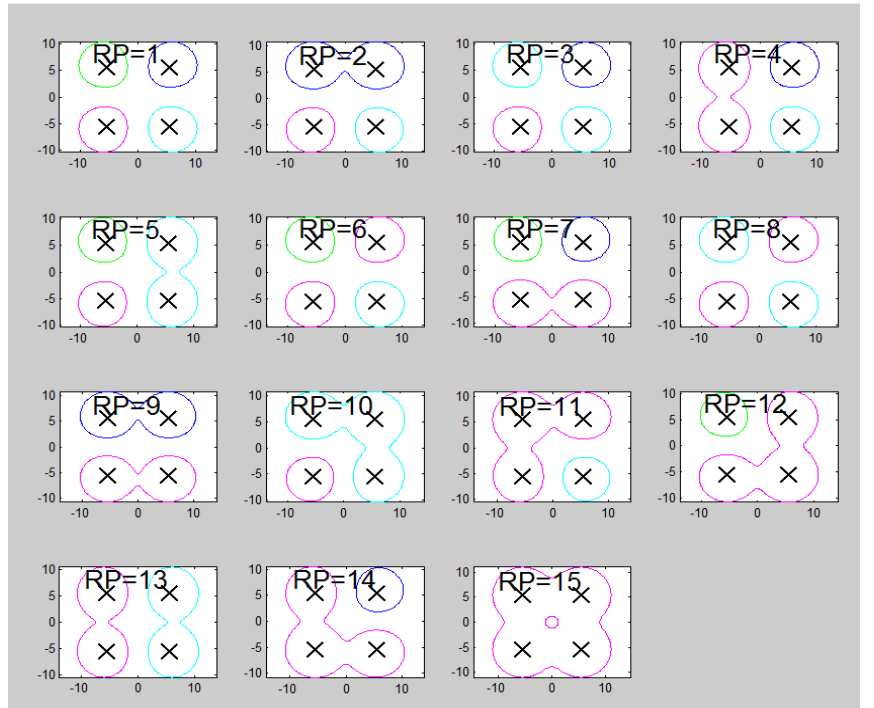

Fig. 4. There are 15 possible resource plans in CT-DSFN with 4 transmitters. The calculated coverage of each zone has different color. (No fading.)

\section{B. Three categories of resource plan sets}

An objective of this study is to compare the following three categories of resource plan sets:

\section{Scheme E: Continuous transmission DSFN}

This case is illustrated in Fig. 2 and 4. All transmitters are continuously transmitting at full power $P_{\max }$, i.e. all columns of all transmitter assignment plans contain at least one nonzero element.

$$
\sum_{z} T_{z, i}^{(r)}>0, \quad \forall z, r
$$

Our example: This corresponds to only utilizing resource plan 1 and 4 in Fig. 3, and in the set $\mathrm{T}$ in equation (8).

\section{Scheme F: No SFN}

Maximum one transmitter is sending in each zone, i.e. there is maximum one non-zero element of each row of each of the transmitter assignment plans:

$$
\sum_{i} \theta\left(T_{z, i}^{(r)}\right) \leq 1, \quad \forall z, r .
$$

Our example: This corresponds to only utilizing resource plan 1 and 4 in Fig. 3, and in the set $\mathrm{T}$ in (8).

\section{Scheme G: Non-continuous transmission DSFN}

This case can be described as a union of the resource plan sets of category (i) and (ii). Some transmitters are blocked in some resource plans, such that at least one column of one transmitter assignment plan only consist of zero elements. Some transmitters are grouped into SFNs of size at least two, i.e. at least of one the rows in one of the plans consist of two or more non-zero elements.

Our example: This corresponds to using all four plans in Fig. 3 , and in the set $\mathbf{T}$ in (8).

\section{RESULTS}

The set of all possible resource plans that fulfills criteria (8) to (11) for each of the above three PARPS schemes is generated, and performance is evaluated.

\section{A. Scalability analysis}

In this section, the number of possible resource plans that fulfills the criteria in section B are analyzed. Fig. 4 illustrates that the number of possible resource plans in scheme E (CTDSFN) with 4 transmitters is 15 . The number of zones is 4 in the first resource plan, and less in the others. A full PARPS scheduling scheme, with one queue per zone in each resource plan, would require 37 queues in this case. Many of them are however not very efficient, and would not be used by the scheduling scheme. For example, resource plan 8 only offers two zones (two simultaneous data streams) and poor coverage.

TABLE II

NUMBER OF POSSIBLE UNIQUE RESOURCE PLANS

\begin{tabular}{|l||r|r|r|r|r|r|r|r|c|} 
& $\left.\right|_{\mathrm{Tx}}=1$ & 2 & 3 & 4 & 5 & 6 & 7 & 8 & $\mathrm{~N}$ \\
\hline \hline $\begin{array}{l}\text { F. No } \\
\text { SFN }\end{array}$ & 1 & 3 & 7 & 15 & 31 & 63 & 127 & 255 & $2^{N}-1$ \\
\hline $\begin{array}{l}\text { E. CT- } \\
\text { DSFN }\end{array}$ & 1 & 2 & 5 & 15 & 52 & 203 & 877 & 4140 & $B_{N}$ \\
\hline $\begin{array}{l}\text { G. NCT- } \\
\text { DSFN }\end{array}$ & 1 & 4 & 14 & 51 & 202 & 876 & 4139 & 21146 & $B_{N+1^{-}}$ \\
\hline \hline
\end{tabular}

Table II shows that the number of possible resource plans of scheme $\mathrm{E}, \mathrm{F}$ and $\mathrm{G}$ respectively grows rapidly with the number of centrally controlled transmitters.

It can be observed that, in the case of no SFN, the number of possible resource plans is $2^{N_{T X}}-1$. It can also be observed that the number of DSFN resource plans grows according to the Bell numbers $B_{N}$, which is the number of ways a set of $N$ elements can be partitioned. It has been shown that the Bell numbers can be calculated using a recursive formula (starting with $B_{0}=B_{I}=1$ ): [10]

$$
B_{n+1}=\sum_{k=0}^{n}\left(\begin{array}{l}
n \\
k
\end{array}\right) B_{k}
$$

The number of possible resource plans grows nonpolynomially with the size $N_{\mathrm{Tx}}$ of the system. The computational complexity of PARPS grows at least linearly with the number of resource plans. Using the complete set of possible plans would not scale to large system.

However, the complete set of possible plans is of interest for a theoretical analysis of the maximum performance bounds, for example to compare strategy E, F and G.

\section{B. Effect of using a subset of the resource plans}

In practical systems, the set of resource plans should be limited to the most frequently used plans, due to the scalability issue. In the small case studied in Fig. 5, if only $10 \%$ of the numbers of theoretically possible resource plans are utilized, the MSSE performance is only reduced by $17 \%$. This is an indication that the problem size does not have to grow as fast as in table II, if a performance loss can be accepted. 


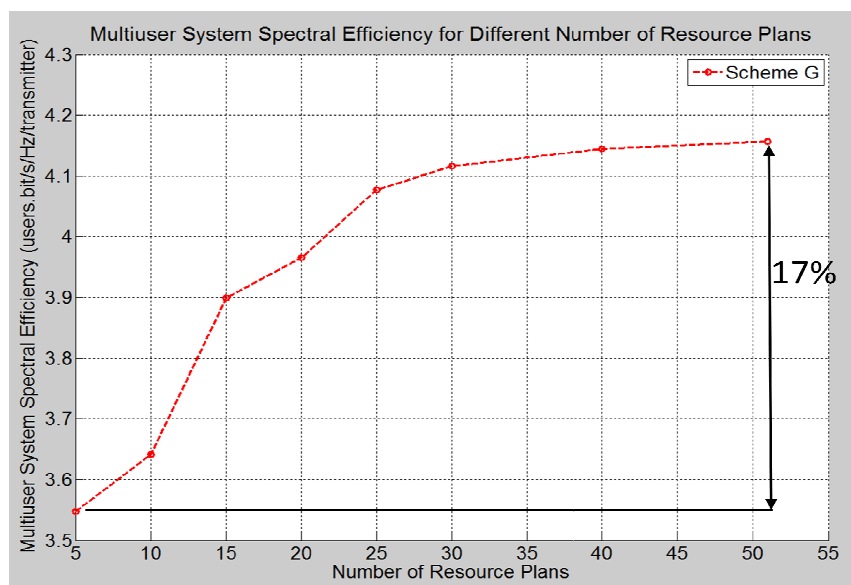

Fig. 5. If only the 5 most frequent resource plans are used, out of the 51 possible plans, the MSSE performance is only deteriorated by $17 \%$. (For scheme $\mathrm{G}$ with 4 transmitters.)

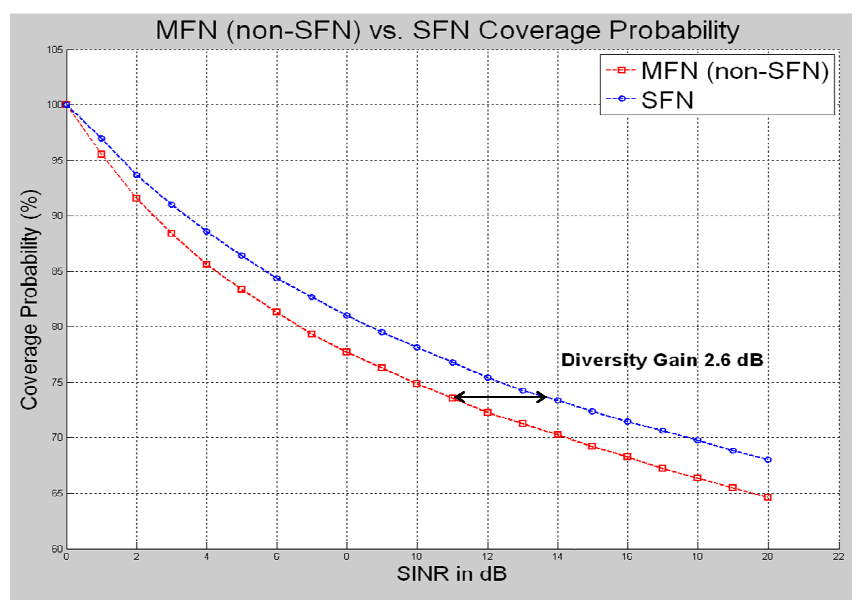

Fig. 6. Coverage probability (y axis) for non-SFN (scheme A, B and F) and SFN (scheme $\mathrm{C}, \mathrm{D}, \mathrm{E}$ and $\mathrm{G}$ ), vs signal-to-interference-and-noise ratio requirement of the applied modulation and coding scheme ( $\mathrm{x}$ axis).

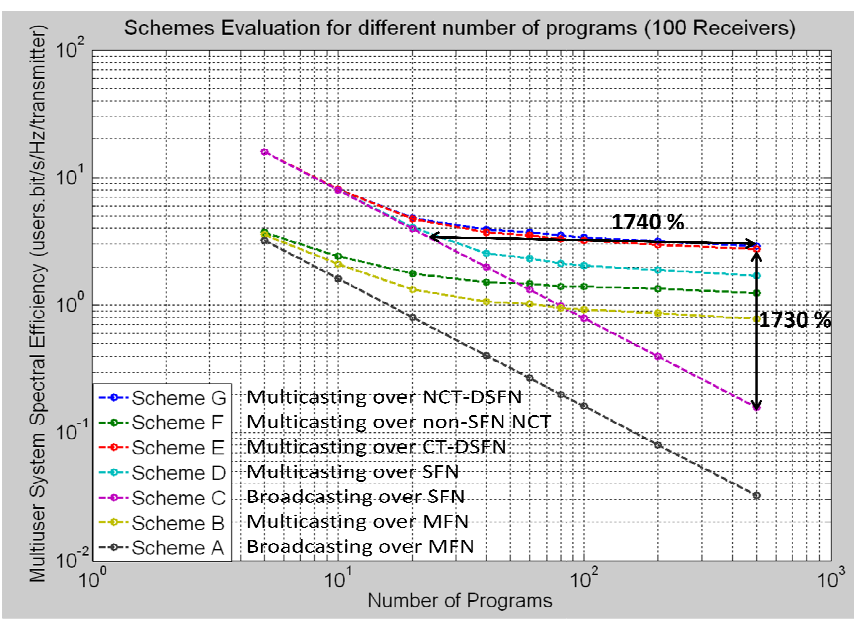

Fig. 7. Multiuser Spectral Efficiency (MSSE) for each scheme, for different numbers of offered programs, 100 receivers, 4 transmitters, and the heterogeneous user behavior model.
TABLE III

MSSE PERFORMANCE IMPROVEMENT FOR 100 OFFERED TV PROGRAMS AND 100 RECEIVERS

\begin{tabular}{clccc}
$\begin{array}{c}\text { Number of } \\
\text { transmitters }\end{array}$ & $\begin{array}{l}\text { User } \\
\text { behavior }\end{array}$ & $\begin{array}{l}\text { Scheme D } \\
\text { vs. C }\end{array}$ & $\begin{array}{l}\text { Scheme E } \\
\text { vs. C }\end{array}$ & $\begin{array}{l}\text { Scheme G } \\
\text { vs. C }\end{array}$ \\
\hline \hline 3 & $\begin{array}{l}\text { Hetero- } \\
\text { geneous }\end{array}$ & $193 \%$ & $321 \%$ & $342 \%$ \\
4 & $\begin{array}{l}\text { Hetero- } \\
\text { geneous }\end{array}$ & $156 \%$ & $302 \%$ & $329 \%$ \\
4 & $\begin{array}{l}\text { Homo- } \\
\text { geneous }\end{array}$ & $132 \%$ & $309 \%$ & $335 \%$ \\
\hline
\end{tabular}

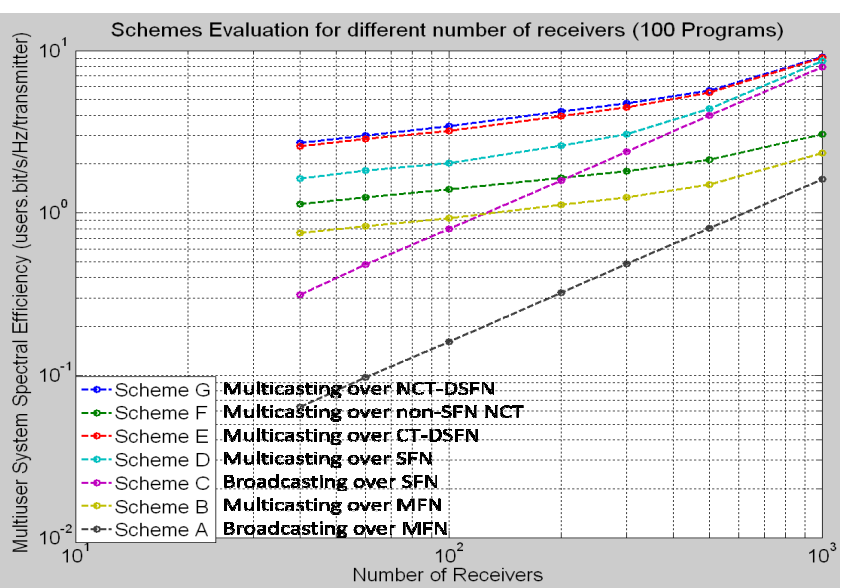

Fig. 8. Multiuser System Spectral Efficiency (MSSE) for each scheme, for different numbers of receivers, 100 offered programs, 4 transmitters, and the heterogeneous user behavior model.

\section{Diversity gain}

As seen from the Fig. 6, SFN gives a diversity gain of $2.6 \mathrm{~dB}$ for 4 transmitters, which according to Shannon-Heartly allows an additional $23 \%$ increase in information rate for the same coverage for the simulated case. In the results below, this information rate increase is assumed, and the same coverage probability of all schemes, making their SSE figures comparable. The diversity gain increases with the number coordinated transmitters.

\section{Spectral efficiency}

Performance in terms of Multiuser System Spectral Efficiency (MSSE) for several parameter cases are presented in Fig. 7 and 8 , and in Table III, for different parameter cases. Since the number of active receivers (or users) are constant in fig. 7 and in table III, the SSE is proportional to the MSSE.

Scheme D shows up to $970 \%$ gain improvement in SSE and MSSE over scheme $\mathrm{C}$ for the simulated cases, meaning that the same amount of TV programs can be offered using in average only $9 \%$ of the spectrum. Scheme E shows up to $1730 \%$ improvement in SSE and MSSE over scheme C. Scheme E allows up to $1740 \%$ more TV programs than scheme $\mathrm{C}$ in average over the same spectrum for the same amount of receivers. 


\section{CONCLUSIONS}

An emerging struggle can be discerned for the attractive UHF band frequencies that have been released from analog TV transmission in many countries. Spectrum efficiency is a key factor in technical comparisons of wireless standards. The success of DVB-T2 in frequency regulation decisions and in frequency auctions may depend on if its spectral efficiency can be made comparable with state-of-the-art cellular systems competing for the same frequency bands.

Our results suggest that interactive multicasting (scheme D) is highly efficient in terms of system spectral efficiency (SSE), meaning a higher number of offered TV programs over the same spectrum. They also suggest increase in multiuser system spectral efficiency (MSSE), implying higher number of happy viewers. Even better results can be achieved by combining interactive multicasting with DSFN (scheme E). Such SFN based solutions also allow better coverage than non-SFN solutions.

An unexpected result is that no significant further improvement is achieved if utilizing non-continuous transmission DSFN (scheme G). Also, feasibility of noncontinuous transmission (scheme $\mathrm{F}$ and $\mathrm{G}$ ) remains to be seen, as today's DVB-T2 equipment do not support on-off switching of the transmitted signal as yet.

The results are further increasing with the number of coordinated transmitters, and also increasing with the number of offered TV programs, but decreasing with the number of receivers per transmitter. The suggested schemes are therefore especially of interest for low-power transmitters for mobileTV. The suggested heterogeneous user-behavior model for program selection results in better improvement than a homogeneous model.

Implementing multicasting over continuous transmission DSFN is feasible with the current DVB-T2 infrastructure; timeslots are enabled by time slicing.

A major contribution of this paper is systematic approach to analyzing the upper bound for the performance that is possible for each of the three schemes E, F, and G. This is done by generating all possible PARPS resource plans for these cases.

Scalability is analyzed by deriving analytical expressions for the number of possible resource plans. A drawback with PARPS is its computational complexity, since the number of possible resource plans grows rapidly with the number of coordinated transmitters. Simulation results however show that a majority of the resource plans can be skipped with little performance loss.

Future research involves improving the computational complexity of DSFN, for example by using the greedy and distributed algorithms suggested in [3], which is scalable to larger networks, but only allows CT-DSFN.

Future research also involves investigating the ability of PARPS to control other radio resource management parameters, such as multiple-input multiple-output (MIMO) transmission, error coding rate and power control. This may potentially be a way to further improve the IP multicast efficiency not only in DVB-T2. It may also be an approach to the LTE 4G cellular system features Coordinated Multi-point (COMP), enhanced Multicast Broadcast Multimedia Service (eMBMS) and Multicast-Broadcast Single Frequency Networks (MB-SFN).

\section{ACKNOWLEDGEMENTS}

This work has been supported by grant 00156702 of the EU European Regional Development Fund, Mellersta Norrland, Sweden, and by grant 00155148 of Länsstyrelsen Västernorrland, Sweden to the Realistic 3D research group at Mid Sweden University.

We also want to thank Mr Ashfaq Malik, who in his M.Sc. thesis made contributions to the simulation model.

\section{REFERENCES}

[1] N. Sinha, R. Oz and S. V. Vasudevan, "The statistics of switched broadcast", Proceedings of the SCTE 2005 Conference on Emerging Technologies, Tampa, FL, USA, January 2005.

[2] M. Eriksson, H. Säterberg, "The concept of PARPS - Packet and Resource Plan Scheduling", October 1999, Venice, Italy

[3] M. Eriksson, Dynamic Single Frequency Networks, IEEE J. on Selected Areas in Communications, vol. 19, no. 10, pp. 1905-1914, Oct 2001.

[4] G. May and P. Unger, "A new approach for transmitting local content within digital single frequency broadcast networks," IEEE Trans. Broadcasting, vol. 53, no. 4, pp. 732-737, Dec. 2007.

[5] J. López-Sánchez, C. Romero, D. Gómez-Barquero, N. Cardona "Optimization of DVB-T Networks for the Provision of Local and Mobile Services," ISSN 1889-8297 / Waves, 2011 page 48-57

[6] Antonios A, Christos B, Vasileios K, Andreas P, George T, "Spectral Efficiency Performance of MBSFN-enabled LTE Networks", 2010 IEEE $6^{\text {th }}$ International Conference on Wireless and Mobile Computing, Networking and Communications.

[7] U. Jennehag, S. Pettersson , "On Synchronization Frames for Channel Switching in a GOP-Based IPTV Environment". CCNC 2008. 5th IEEE January 2008.

[8] G. Malmgren, Single Frequency Broadcasting Networks, $\mathrm{PhD}$ thesis, Dept. of Signals, Sensors and Systems, Royal Inst. of Technology, 1997.

[9] T. S. Rappaport, Wireless communications principles and practices, 2002, Prentice-Hall, p. 102.

[10] H. S. Wilf, Generating functionology (2nd ed.). Boston, MA: Academic Press, 1994.

[11] O. Berglingm Utveckling av tjänsteportal för MHP-plattformen. 2007. $\mathrm{PhD}$ Thesis. University of Gävle. 